\title{
Linear equations with unknowns from a multiplicative group in a function field
}

by

Jan-Hendrik Evertse (Leiden) and Umberto Zannier (Pisa)

To Professor Wolfgang Schmidt on his 75th birthday

1. Introduction. Let $K$ be a field of characteristic 0 , and $n$ an integer $\geq 2$. Denote by $\left(K^{*}\right)^{n}$ the $n$-fold direct product of the multiplicative group $K^{*}$. Thus, the group operation of $\left(K^{*}\right)^{n}$ is coordinatewise multiplication $\left(x_{1}, \ldots, x_{n}\right) \cdot\left(y_{1}, \ldots, y_{n}\right)=\left(x_{1} y_{1}, \ldots, x_{n} y_{n}\right)$. We write $\left(x_{1}, \ldots, x_{n}\right)^{m}:=$ $\left(x_{1}^{m}, \ldots, x_{n}^{m}\right)$ for $m \in \mathbb{Z}$. We will often denote elements of $\left(K^{*}\right)^{n}$ by bold face characters $\mathbf{x}, \mathbf{y}$, etc.

Evertse, Schlickewei and Schmidt [3] proved that if $\Gamma$ is a subgroup of $\left(K^{*}\right)^{n}$ of finite rank $r$ and $a_{1}, \ldots, a_{n}$ are non-zero elements of $K$, then the equation

$$
a_{1} x_{1}+\cdots+a_{n} x_{n}=1 \quad \text { in } \mathbf{x}=\left(x_{1}, \ldots, x_{n}\right) \in \Gamma
$$

has at most $e^{(6 n)^{3 n}(r+1)}$ non-degenerate solutions, i.e., solutions with

$$
\sum_{i \in I} a_{i} x_{i} \neq 0 \quad \text { for each proper, non-empty subset } I \text { of }\{1, \ldots, n\} .
$$

In the present paper, we derive a function field analogue of this result. Thus, let $k$ be an algebraically closed field of characteristic 0 and let $K$ be a transcendental field extension of $k$, where we allow the transcendence degree to be arbitrarily large. Let $\Gamma$ be a subgroup of $\left(K^{*}\right)^{n}$ such that $\left(k^{*}\right)^{n} \subset \Gamma$ and such that $\Gamma /\left(k^{*}\right)^{n}$ has finite rank. This means that there are $\mathbf{a}_{1}, \ldots, \mathbf{a}_{r} \in \Gamma$ such that for every $\mathbf{x} \in \Gamma$ there are integers $m, z_{1}, \ldots, z_{r}$ with $m>0$ and $\xi \in\left(k^{*}\right)^{n}$ such that $\mathbf{x}^{m}=\xi \cdot \mathbf{a}_{1}^{z_{1}} \cdots \mathbf{a}_{r}^{z_{r}}$. If $\Gamma=\left(k^{*}\right)^{n}$ then $\Gamma /\left(k^{*}\right)^{n}$ has rank 0; otherwise, $\operatorname{rank}\left(\Gamma /\left(k^{*}\right)^{n}\right)$ is the smallest $r$ for which there exist $\mathbf{a}_{1}, \ldots, \mathbf{a}_{r}$ as above. 
We deal again with equation (1.1) in solutions $\left(x_{1}, \ldots, x_{n}\right) \in \Gamma$ with coefficients $a_{1}, \ldots, a_{n} \in K^{*}$. We mention that in the situation we are considering now, (1.1) might have infinitely many non-degenerate solutions. But one can show that the set of non-degenerate solutions of (1.1) is contained in finitely many $\left(k^{*}\right)^{n}$-cosets, i.e., in finitely many sets of the shape $\mathbf{b} \cdot\left(k^{*}\right)^{n}=\left\{\mathbf{b} \cdot \xi: \xi \in\left(k^{*}\right)^{n}\right\}$ with $\mathbf{b} \in \Gamma$. More precisely, we prove the following:

THEOREM. Let $k$ be an algebraically closed field of characteristic 0 , let $K$ be a transcendental extension of $k$, let $n \geq 2$, let $a_{1}, \ldots, a_{n} \in K^{*}$, and let $\Gamma$ be a subgroup of $\left(K^{*}\right)^{n}$ satisfying

$$
\left(k^{*}\right)^{n} \subset \Gamma, \quad \operatorname{rank}\left(\Gamma /\left(k^{*}\right)^{n}\right)=r .
$$

Then the set of non-degenerate solutions of equation (1.1) is contained in the union of not more than

$$
\sum_{i=2}^{n+1}\left(\begin{array}{l}
i \\
2
\end{array}\right)^{r}-n+1
$$

$\left(k^{*}\right)^{n}$-cosets.

We mention that Bombieri, Mueller and Zannier [1] by means of a new approach gave a rather sharp upper bound for the number of solutions of polynomial-exponential equations in one variable over function fields. Their approach and result were extended by Zannier [5] to polynomial-exponential equations over function fields in several variables. Our proof heavily uses the arguments from this last paper.

Let us consider the case $n=2$, that is, let us consider the equation

$$
a_{1} x_{1}+a_{2} x_{2}=1 \quad \text { in }\left(x_{1}, x_{2}\right) \in \Gamma,
$$

where $\Gamma, a_{1}, a_{2}$ satisfy the hypotheses of the Theorem with $n=2$. It is easy to check that all solutions $\left(x_{1}, x_{2}\right)$ of $(1.5)$ with $a_{1} x_{1} / a_{2} x_{2} \in k^{*}$ (if any such exist) lie in the same $\left(k^{*}\right)^{2}$-coset, while any two different solutions $\left(x_{1}, x_{2}\right)$ with $a_{1} x_{1} / a_{2} x_{2} \notin k^{*}$ lie in different $\left(k^{*}\right)^{2}$-cosets. So our Theorem implies that (1.5) has at most $3^{r}$ solutions $\left(x_{1}, x_{2}\right)$ with $a_{1} x_{1} / a_{2} x_{2} \notin k^{*}$. This is a slight extension of a result by Zannier [5] who obtained the same upper bound, but for groups $\Gamma=\Gamma_{1} \times \Gamma_{1}$ where $\Gamma_{1}$ is a subgroup of $K^{*}$.

The formulation of our Theorem was inspired by Mueller [4]. She proved that if $S$ is a finite set of places of the rational function field $k(z)$, if $\Gamma=U_{S}^{n}$ is the $n$-fold direct product of the group of $S$-units in $k(z)^{*}$, and if $a_{1}, \ldots, a_{n} \in$ $k(z)^{*}$, then the set of non-degenerate solutions of (1.1) is contained in the union of not more than $(e(n+1) ! / 2)^{n(2|S|+1)}\left(k^{*}\right)^{n}$-cosets.

Evertse and Györy [2] also considered equation (1.1) with $\Gamma=U_{S}^{n}$, but in the more general situation that $S$ is a finite set of places in any finite extension $K$ of $k(z)$. They showed that if $K$ has genus $g$ and if $a_{1}, \ldots, a_{n}$ 
$\in K^{*}$ then the set of solutions $\mathbf{x} \in U_{S}^{n}$ of $(1.1)$ with $\left(a_{1} x_{1}, \ldots, a_{n} x_{n}\right) \notin\left(k^{*}\right)^{n}$ is contained in the union of not more than

$$
\log (g+2) \cdot(e(n+1))^{(n+1)|S|+2}
$$

proper linear subspaces of $K^{n}$.

We mention that in general $\operatorname{rank} U_{S}^{n} \leq n(|S|-1)$ but that in contrast to number fields, equality need not hold. From our Theorem we can deduce the following result, which removes the dependence on the genus $g$, and replaces the dependence on $|S|$ by one on the rank.

Corollary. Let $k, K, n, a_{1}, \ldots, a_{n}, \Gamma, r$ be as in the Theorem. Then the set of solutions of $(1.1)$ with $\left(a_{1} x_{1}, \ldots, a_{n} x_{n}\right) \notin\left(k^{*}\right)^{n}$ is contained in the union of not more than

$$
\sum_{i=2}^{n+1}\left(\begin{array}{l}
i \\
2
\end{array}\right)^{r}+2^{n}-2 n-1
$$

proper linear subspaces of $K^{n}$.

In Section 2 we prove some auxiliary results for formal power series, in Section 3 we prove our Theorem in the case that $K$ has transcendence degree 1 over $k$, in Section 4 we extend this to the general case that $K$ is an arbitrary transcendental extension of $k$, and in Section 5 we deduce the Corollary.

2. Results for formal power series. Let $k$ be an algebraically closed field of characteristic 0 . Let $z$ be an indeterminate. Denote as usual by $k[[z]]$ the ring of formal power series over $k$ and by $k((z))$ its quotient field. Thus, $k((z))$ consists of series $\sum_{i \geq i_{0}} c_{i} z^{i}$ with $i_{0} \in \mathbb{Z}$ and $c_{i} \in k$ for $i \geq i_{0}$. We endow $k((z))$ with a derivation $\frac{d}{d z}: \sum_{i \geq i_{0}} c_{i} z^{i} \mapsto \sum_{i \geq i_{0}} i c_{i} z^{i-1}$. Let $1+z k[[z]]$ denote the set of all formal power series of the shape $1+c_{1} z+$ $c_{2} z^{2}+\cdots$ with $c_{1}, c_{2}, \ldots \in k$. Clearly, $1+z k[[z]]$ is a multiplicative group. For $f \in 1+z k[[z]], u \in k$ we define

$$
f^{u}:=\sum_{i=0}^{\infty}\left(\begin{array}{l}
u \\
i
\end{array}\right)(f-1)^{i},
$$

where $\left(\begin{array}{l}u \\ 0\end{array}\right)=1$ and $\left(\begin{array}{l}u \\ i\end{array}\right)=u(u-1) \cdots(u-i+1) / i$ ! for $i>0$. Thus, $f^{u}$ is a well-defined element of $1+z k[[z]]$. This definition of $f^{u}$ coincides with the usual one for $u=0,1,2, \ldots$ We have $\frac{d}{d z} f^{u}=u f^{u-1} \frac{d}{d z} f$, and moreover,

$$
\begin{cases}(f g)^{u}=f^{u} g^{u} & \text { for } f, g \in 1+z k[[z]], u \in k ; \\ f^{u+v}=f^{u} f^{v} \text { and }\left(f^{u}\right)^{v}=f^{u v} & \text { for } f \in 1+z k[[z]], u, v \in k .\end{cases}
$$

(One may verify (2.2) by taking logarithmic derivatives and recalling that two series in $1+z k[[z]]$ are equal if and only if their logarithmic derivatives 
are equal.) We endow $(1+z k[[z]])^{r}$ with the usual coordinatewise multiplication. Given $\mathbf{B}=\left(b_{1}, \ldots, b_{r}\right) \in(1+z k[[z]])^{r}$, we define $\mathbf{B}^{u}:=\left(b_{1}^{u}, \ldots, b_{r}^{u}\right)$ for $u \in k$ and $\mathbf{B}^{\mathbf{u}}:=b_{1}^{u_{1}} \cdots b_{r}^{u_{r}}$ for $\mathbf{u}=\left(u_{1}, \ldots, u_{r}\right) \in k^{r}$. Thus, $\mathbf{B}^{u} \in$ $(1+z k[[z]])^{r}$ and $\mathbf{B}^{\mathbf{u}} \in 1+z k[[z]]$.

Let $h, r$ be integers with $h \geq 2, r \geq 1$. Further, let $a_{1}, \ldots, a_{h}$ be elements of $k[[z]]$ which are algebraic over the field of rational functions $k(z)$ and which are not divisible by $z$, and let $\alpha_{i j}(i=1, \ldots, h, j=1, \ldots, r)$ be elements of $1+z k[[z]]$ which are algebraic over $k(z)$. Put $\mathbf{A}_{i}:=\left(\alpha_{i 1}, \ldots, \alpha_{i r}\right)$ $(i=1, \ldots, h)$. Define

$$
R:=\left\{\mathbf{u} \in k^{r}: a_{1} \mathbf{A}_{1}^{\mathbf{u}}, \ldots, a_{h} \mathbf{A}_{h}^{\mathbf{u}} \text { are linearly dependent over } k\right\} .
$$

By a class we mean a set $R^{\prime} \subset k^{r}$ with the property that there are a subset $J$ of $\{1, \ldots, h\}$ and $\mathbf{u}_{0} \in \mathbb{Q}^{r}$ such that for every $\mathbf{u} \in R^{\prime}$,

$$
\left\{\begin{array}{l}
a_{i} \mathbf{A}_{i}^{\mathbf{u}}(i \in J) \text { are linearly dependent over } k ; \\
\left(\mathbf{A}_{i} \mathbf{A}_{j}^{-1}\right)^{\mathbf{u}-\mathbf{u}_{0}}=1 \text { for all } i, j \in J .
\end{array}\right.
$$

LEMMA $1 . R$ is the union of finitely many classes.

Proof. This is basically a special case of [5, Lemma 1]. In the proof of that lemma, it was assumed that $k=\mathbb{C}$, and that the $a_{i}$ and $\alpha_{i j}$ are holomorphic functions in the variable $z$ which are algebraic over $\mathbb{C}(z)$ and which are defined and have no zeros on a simply connected open subset $\Omega$ of $\mathbb{C}$. It was shown that provided $k=\mathbb{C}$, this was no loss of generality. The argument remains precisely the same if one allows $k$ to be an arbitrary algebraically closed field of characteristic 0 and if one takes for the $a_{i}$ power series from $k[[z]]$ which are algebraic over $k(z)$ and which are not divisible by $z$, and for the $\alpha_{i j}$ power series from $1+z k[[z]]$ which are algebraic over $k(z)$.

We mention that in [5] the definition of a class is slightly different

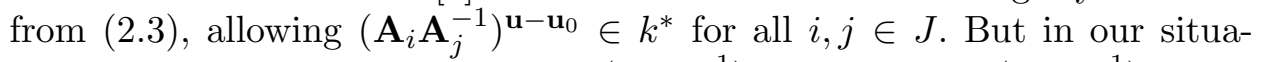
tion this implies automatically that $\left(\mathbf{A}_{i} \mathbf{A}_{j}^{-1}\right)^{\mathbf{u}-\mathbf{u}_{0}}=1$ since $\left(\mathbf{A}_{i} \mathbf{A}_{j}^{-1}\right)^{\mathbf{u}-\mathbf{u}_{0}} \in$ $1+z k[[z]]$

We now impose some further restriction on the $\alpha_{i j}$ and prove a more precise result. Namely, we assume that

$$
\left\{\mathbf{u} \in k^{r}:\left(\mathbf{A}_{i} \cdot \mathbf{A}_{h}^{-1}\right)^{\mathbf{u}}=1 \text { for } i=1, \ldots, h\right\}=\{\mathbf{0}\} .
$$

Let $S$ be the set of $\mathbf{u} \in k^{r}$ such that there are $\xi_{1}, \ldots, \xi_{h} \in k$ with

$$
\sum_{i=1}^{h} \xi_{i} a_{i} \mathbf{A}_{i}^{\mathbf{u}}=0
$$

$$
\sum_{i \in I} \xi_{i} a_{i} \mathbf{A}_{i}^{\mathbf{u}} \neq 0 \quad \text { for each proper, non-empty subset } I \text { of }\{1, \ldots, h\} .
$$

Lemma 2. Assume (2.4). Then $S$ is finite. 
Proof. We prove a slightly stronger statement. We partition $\{1, \ldots, h\}$ into subsets $I_{1}, \ldots, I_{s}$ such that $\mathbf{A}_{i}=\mathbf{A}_{j}$ if and only if $i, j$ belong to the same set $I_{l}$ for some $l \in\{1, \ldots, s\}$. Let $\widetilde{S}$ be the set of $\mathbf{u} \in k^{r}$ for which there exist $\xi_{1}, \ldots, \xi_{n} \in k$ such that (2.5) holds and, instead of (2.6),

$$
\sum_{i \in I} \xi_{i} a_{i} \mathbf{A}_{i}^{\mathbf{u}} \neq 0
$$

holds for each proper, non-empty subset $I$ of $\{1, \ldots, h\}$ which is a union of some of the sets $I_{1}, \ldots, I_{s}$. We prove that $\widetilde{S}$ is finite. This clearly suffices.

We proceed by induction on $p:=h+s$. Notice that from assumption (2.4) it follows that $h \geq 2$ and $s \geq 2$. First let $h=2, s=2$, i.e., $p=4$. Thus, $\widetilde{S}$ is the set of $\mathbf{u} \in k^{r}$ for which there are non-zero $\xi_{1}, \xi_{2} \in k$ with $\xi_{1} a_{1} \mathbf{A}_{1}^{\mathbf{u}}+\xi_{2} a_{2} \mathbf{A}_{2}^{\mathbf{u}}=0$. Then for $\mathbf{u} \in \widetilde{S}$ we have

$$
\left(\mathbf{A}_{1} \cdot \mathbf{A}_{2}^{-1}\right)^{\mathbf{u}}=\xi\left(a_{2} a_{1}^{-1}\right)
$$

with $\xi \in k^{*}$. Consequently, $\left(\mathbf{A}_{1} \cdot \mathbf{A}_{2}^{-1}\right)^{\mathbf{u}_{2}-\mathbf{u}_{1}} \in k^{*}$ for any $\mathbf{u}_{1}, \mathbf{u}_{2} \in \widetilde{S}$. But then for $\mathbf{u}_{1}, \mathbf{u}_{2} \in \widetilde{S}$ we must have $\left(\mathbf{A}_{1} \cdot \mathbf{A}_{2}^{-1}\right)^{\mathbf{u}_{2}-\mathbf{u}_{1}}=1$ since $\left(\mathbf{A}_{1} \cdot \mathbf{A}_{2}^{-1}\right)^{\mathbf{u}_{2}-\mathbf{u}_{1}} \in$ $1+z k[[z]]$. In view of assumption $(2.4)$ this implies that $\widetilde{S}$ consists of at most one element.

Now let $p>4$ and assume Lemma 2 to be true for all pairs $(h, s)$ with $h \geq 2, s \geq 2$ and $h+s<p$. We apply Lemma 1 above. Clearly, $\widetilde{S}$ is contained in the set $R$ dealt with in Lemma 1, and therefore, $\widetilde{S}$ is the union of finitely many sets $\widetilde{S} \cap R^{\prime}$ where $R^{\prime}$ is a class as in (2.3). So we have to show that each such set $\widetilde{S} \cap R^{\prime}$ is finite.

Thus let $S^{\prime}:=\widetilde{S} \cap R^{\prime}$, where $R^{\prime}$ is a typical one among these sets. Let $J$ be the corresponding subset of $\{1, \ldots, h\}$, and $\mathbf{u}_{0} \in \mathbb{Q}^{r}$ the corresponding vector, such that (2.3) holds. We distinguish two cases. First suppose that $J$ is contained in some set $I_{l}$. Then the elements $a_{j}(j \in J)$ are linearly dependent over $k$. There is a proper subset $J^{\prime}$ of $J$ such that $a_{j}\left(j \in J^{\prime}\right)$ are linearly independent over $k$ and such that each $a_{j}$ with $j \in J \backslash J^{\prime}$ can be expressed as a linear combination over $k$ of the $a_{j}$ with $j \in J^{\prime}$. By substituting these linear combinations into (2.5), (2.7), we obtain similar conditions, but with $I_{l}$ replaced by the smaller set obtained by removing from $I_{l}$ the elements from $J \backslash J^{\prime}$. This reduces the number $h$ of terms. Further, condition (2.4) remains valid. Thus we may apply the induction hypothesis, and conclude that $S^{\prime}$ is finite.

Now assume that $J$ is not contained in one of the sets $I_{l}$. We transform our present situation into a new one with instead of $I_{1}, \ldots, I_{s}$ a partition of $\{1, \ldots, h\}$ into fewer than $s$ sets. Then again, the induction hypothesis is applicable. 
There are $i, j \in J$ with $\mathbf{A}_{i} \neq \mathbf{A}_{j}$, say $i \in I_{l_{1}}$ and $j \in I_{l_{2}}$. Further, there is $\mathbf{u}_{0} \in \mathbb{Q}^{r}$ such that $\left(\mathbf{A}_{i} \mathbf{A}_{j}^{-1}\right)^{\mathbf{u}-\mathbf{u}_{0}}=1$ for $\mathbf{u} \in S^{\prime}$. According to an argument in the proof of Lemma 1 of [5], the set of $\mathbf{u} \in k^{r}$ with $\left(\mathbf{A}_{i} \mathbf{A}_{j}^{-1}\right)^{\mathbf{u}}=1$ is a linear subspace $V$ of $k^{r}$ which is defined over $\mathbb{Q}$. Let $\mathbf{v}_{1}, \ldots, \mathbf{v}_{r^{\prime}}$ be a basis of $V$ contained in $\mathbb{Z}^{r}$. Thus, each $\mathbf{u} \in S^{\prime}$ can be expressed uniquely as

$$
\mathbf{u}_{0}+w_{1} \mathbf{v}_{1}+\cdots+w_{r^{\prime}} \mathbf{v}_{r^{\prime}} \quad \text { with } \mathbf{w}=\left(w_{1}, \ldots, w_{r^{\prime}}\right) \in k^{r^{\prime}} .
$$

Now define

$$
b_{q}:=a_{q} \mathbf{A}_{q}^{\mathbf{u}_{0}}, \quad \mathbf{B}_{q}:=\left(\mathbf{A}_{q}^{\mathbf{v}_{1}}, \ldots, \mathbf{A}_{q}^{\mathbf{v}_{r^{\prime}}}\right) \quad(q=1, \ldots, h) .
$$

Thus, for $\mathbf{u} \in S^{\prime}$ we have

$$
a_{q} \mathbf{A}_{q}^{\mathbf{u}}=b_{q} \mathbf{B}_{q}^{\mathbf{w}} \quad \text { for } q=1, \ldots, h .
$$

Clearly, $b_{q} \in k[[z]]$ and the coordinates of $\mathbf{B}_{q}$ belong to $1+z k[[z]]$, for $q=$ $1, \ldots, h$. Further, $b_{q}$ and the coordinates of $\mathbf{B}_{q}(q=1, \ldots, h)$ are algebraic over $k(z)$ since $\mathbf{u}_{0} \in \mathbb{Q}^{r}$ and since $\mathbf{v}_{1}, \ldots, \mathbf{v}_{r^{\prime}} \in \mathbb{Z}^{r}$.

From the definition of $\mathbf{B}_{q}(q=1, \ldots, h)$ it follows that if $\left(\mathbf{B}_{q} \mathbf{B}_{h}^{-1}\right)^{\mathbf{w}}=1$ for $q=1, \ldots, h$, then $\left(\mathbf{A}_{q} \mathbf{A}_{h}^{-1}\right)^{\sum_{j} w_{j} \mathbf{v}_{j}}=1$ for $q=1, \ldots, h$, which by (2.4) implies $\sum_{j} w_{j} \mathbf{v}_{j}=\mathbf{0}$ and so $\mathbf{w}=\mathbf{0}$. Therefore, condition (2.4) remains valid if we replace $\mathbf{A}_{q}$ by $\mathbf{B}_{q}$ for $q=1, \ldots, h$.

It is important to notice that $\mathbf{B}_{q_{1}}=\mathbf{B}_{q_{2}}$ for any $q_{1}, q_{2} \in I_{l_{1}} \cup I_{l_{2}}$. Further, for each $l \neq l_{1}, l_{2}$, we have $\mathbf{B}_{q_{1}}=\mathbf{B}_{q_{2}}$ for any $q_{1}, q_{2} \in I_{l}$.

Lastly, if $\mathbf{u} \in S^{\prime}$ then by substituting (2.9) into (2.5), (2.7), we deduce that there are $\xi_{1}, \ldots, \xi_{h} \in k^{*}$ such that $\sum_{q=1}^{h} \xi_{q} b_{q} \mathbf{B}_{q}^{\mathbf{w}}=0$ and $\sum_{q \in I} \xi_{q} b_{q} \mathbf{B}_{q}^{\mathbf{w}}$ $\neq 0$ for each proper subset $I$ of $\{1, \ldots, h\}$ which is a union of some of the sets from $I_{l_{1}} \cup I_{l_{2}}, I_{l}\left(l=1, \ldots, s, l \neq l_{1}, l_{2}\right)$. Thus, each $\mathbf{u} \in S^{\prime}$ corresponds by means of (2.8) to $\mathbf{w} \in k^{r^{\prime}}$ which satisfies similar conditions as $\mathbf{u}$, but with the partition $I_{1}, \ldots, I_{s}$ of $\{1, \ldots, h\}$ replaced by a partition consisting of only $s-1$ sets. Now by the induction hypothesis, the set of $\mathbf{w}$ is finite, and therefore, $S^{\prime}$ is finite. This proves Lemma 2.

We now proceed to estimate the cardinality of $S$. We need a few auxiliary results. For any subset $A$ of $k[[z]]$, we denote by $\operatorname{rank}_{k} A$ the cardinality of a maximal $k$-linearly independent subset of $A$. For each subset $I$ of $\{1, \ldots, h\}$ and each integer $t$ with $1 \leq t \leq h-1$, we define the set

$$
V(I, t)=\left\{\mathbf{u} \in k^{r}: \operatorname{rank}_{k}\left\{a_{i} \mathbf{A}_{i}^{\mathbf{u}}: i \in I\right\} \leq t\right\} .
$$

Clearly, $V(I, t)=k^{r}$ if $t \geq|I|$.

Lemma 3. Let $I, t$ be as above and assume that $t<|I|$. Then $V(I, t)$ is the set of common zeros in $k^{r}$ of a system of polynomials in $k\left[X_{1}, \ldots, X_{r}\right]$, each of total degree at most $\left(\begin{array}{c}t+1 \\ 2\end{array}\right)$.

Proof. The vector $\mathbf{u}$ belongs to $V(I, t)$ if and only if each $t+1$-tuple among the functions $a_{i} \mathbf{A}_{i}^{\mathbf{u}}(i \in I)$ is linearly dependent over $k$, that is, if 
and only if for each subset $J=\left\{i_{0}, \ldots, i_{t}\right\}$ of $I$ of cardinality $t+1$, the Wronskian determinant

$$
\operatorname{det}\left(\left(\frac{d}{d z}\right)^{i} a_{i_{j}} \mathbf{A}_{i_{j}}^{\mathbf{u}}\right)_{i, j=0, \ldots, t}
$$

is identically 0 as a function of $z$. By an argument completely similar to that in the proof of Proposition 1 of [5], one shows that the latter condition is equivalent to $\mathbf{u}$ being a common zero of some finite set of polynomials of degree $\leq\left(\begin{array}{c}t+1 \\ 2\end{array}\right)$. This proves Lemma 3 .

Lemma 4. We have $\mathbf{u} \in S$ if and only if

$$
\begin{aligned}
& \operatorname{rank}_{k}\left\{a_{i} \mathbf{A}_{i}^{\mathbf{u}}: i \in I\right\}+\operatorname{rank}_{k}\left\{a_{i} \mathbf{A}_{i}^{\mathbf{u}}: i \notin I\right\} \\
&>\operatorname{rank}_{k}\left\{a_{i} \mathbf{A}_{i}^{\mathbf{u}}: i=1, \ldots, h\right\}
\end{aligned}
$$

for each proper, non-empty subset I of $\{1, \ldots, h\}$.

Proof. First let $\mathbf{u} \in S$. Take a proper, non-empty subset $I$ of $\{1, \ldots, h\}$. From (2.5), (2.6) it follows that there are $\xi_{1}, \ldots, \xi_{h} \in k$ such that

$$
\sum_{i \in I} \xi_{i} a_{i} \mathbf{A}_{i}^{\mathbf{u}}=-\sum_{i \notin I} \xi_{i} a_{i} \mathbf{A}_{i}^{\mathbf{u}} \neq 0
$$

and therefore the $k$-vector spaces spanned by $\left\{a_{i} \mathbf{A}_{i}^{\mathbf{u}}: i \in I\right\},\left\{a_{i} \mathbf{A}_{i}^{\mathbf{u}}: i \notin I\right\}$, respectively, have non-trivial intersection. This implies (2.11).

Now let $\mathbf{u} \in k^{r}$ be such that (2.11) holds for every proper, non-empty subset $I$ of $\{1, \ldots, h\}$. Let $W$ be the vector space of $\xi=\left(\xi_{1}, \ldots, \xi_{h}\right)$ in $k^{h}$ with $\sum_{i=1}^{h} \xi_{i} a_{i} \mathbf{A}_{i}^{\mathbf{u}}=0$. Further, for a proper, non-empty subset $I$ of $\{1, \ldots, h\}$, let $W(I)$ be the vector space of $\xi=\left(\xi_{1}, \ldots, \xi_{h}\right) \in k^{h}$ with $\sum_{i \in I} \xi_{i} a_{i} \mathbf{A}_{i}^{\mathbf{u}}=0$ and $\sum_{i \notin I} \xi_{i} a_{i} \mathbf{A}_{i}^{\mathbf{u}}=0$. Given a proper, non-empty subset $I$ of $\{1, \ldots, h\}$, it follows from (2.11) that there are $\xi_{1}, \ldots, \xi_{h} \in k$ with $\sum_{i \in I} \xi_{i} a_{i} \mathbf{A}_{i}^{\mathbf{u}}=-\sum_{i \notin I} \xi_{i} a_{i} \mathbf{A}_{i}^{\mathbf{u}} \neq 0$; hence $W(I)$ is a proper linear subspace of $W$. It follows that there is $\xi \in W$ with $\xi \notin W(I)$ for each proper, non-empty subset $I$ of $\{1, \ldots, h\}$. This means precisely that $\mathbf{u} \in S$.

Proposition. Assume (2.4). Then $|S| \leq \sum_{p=2}^{h}\left(\begin{array}{l}p \\ 2\end{array}\right)^{r}-h+2$.

Proof. For $t=1, \ldots, h-1$, let $T_{t}=V(\{1, \ldots, h\}, t)$ (that is, the set of $\mathbf{u} \in k^{r}$ with $\left.\operatorname{rank}_{k}\left\{a_{i} \mathbf{A}_{i}^{\mathbf{u}}: i=1, \ldots, h\right\} \leq t\right)$ and let $S_{t}$ be the set of $\mathbf{u} \in S$ such that $\operatorname{rank}_{k}\left\{a_{i} \mathbf{A}_{i}^{\mathbf{u}}: i=1, \ldots, h\right\}=t$. By (2.11), $\operatorname{rank}_{k}\left\{a_{i} \mathbf{A}_{i}^{\mathbf{u}}: i=\right.$ $1, \ldots, h\}<h$, so $S=S_{1} \cup \cdots \cup S_{h-1}$. We show by induction on $t=1, \ldots, h-1$ that

$$
\left|S_{1} \cup \cdots \cup S_{t}\right| \leq \sum_{p=1}^{t}\left(\begin{array}{c}
p+1 \\
2
\end{array}\right)^{r}-t+1 .
$$

Taking $t=h-1$ proves the Proposition. 
First let $t=1$. Let $\mathbf{u}_{1}, \mathbf{u}_{2} \in S_{1}$. Then $\left(a_{i} a_{h}^{-1}\right)\left(\mathbf{A}_{i} \mathbf{A}_{h}^{-1}\right)^{\mathbf{u}_{j}} \in k^{*}$ for $i=$ $1, \ldots, h, j=1,2$, which implies $\left(\mathbf{A}_{i} \mathbf{A}_{h}^{-1}\right) \mathbf{u}_{1}-\mathbf{u}_{2} \in k^{*}$ for $i=1, \ldots, h$. But then $\left(\mathbf{A}_{i} \mathbf{A}_{h}^{-1}\right)^{\mathbf{u}_{1}-\mathbf{u}_{2}}=1$ since $\left(\mathbf{A}_{i} \mathbf{A}_{h}^{-1}\right)^{\mathbf{u}_{1}-\mathbf{u}_{2}} \in 1+z k[[z]]$ for $i=1, \ldots, h$. Now assumption (2.4) gives $\mathbf{u}_{1}=\mathbf{u}_{2}$. So $\left|S_{1}\right|=1$, which implies (2.12) for $t=1$.

Now assume that $2 \leq t \leq h-1$ and that (2.12) is true with $t$ replaced by any number $t^{\prime}$ with $1 \leq t^{\prime}<t$. By Lemma $3, T_{t}$ is an algebraic subvariety of $k^{r}$, being the set of common zeros of a system of polynomials of degree not exceeding $\left(\begin{array}{c}t+1 \\ 2\end{array}\right)$. By the last part of the proof of Proposition 1 of [5], $T_{t}$ has at most $\left(\begin{array}{c}t+1 \\ 2\end{array}\right)^{r}$ irreducible components.

We first show that $T_{t} \backslash S_{t}$ is a finite union of proper algebraic subvarieties of $T_{t}$. Notice that $\mathbf{u} \in T_{t} \backslash S_{t}$ if and only if either $\operatorname{rank}_{k}\left\{a_{i} \mathbf{A}_{i}^{\mathbf{u}}: i=1, \ldots, h\right\} \leq$ $t-1$ or (by Lemma 4) there are a proper, non-empty subset $I$ of $\{1, \ldots, h\}$ and an integer $q$ with $1 \leq q \leq t-1$ such that $\operatorname{rank}_{k}\left\{a_{i} \mathbf{A}_{i}^{\mathbf{u}}: i \in I\right\} \leq q$ and $\operatorname{rank}_{k}\left\{a_{i} \mathbf{A}_{i}^{\mathbf{u}}: i \notin I\right\} \leq t-q$. This means that $T_{t} \backslash S_{t}$ is equal to the union of $T_{t-1}$ and of all sets $V(I, q) \cap V(\{1, \ldots, h\} \backslash I, t-q)$ with $I$ running through the proper, non-empty subsets of $\{1, \ldots, h\}$ and $q$ running through the integers with $1 \leq q \leq t-1$. By Lemma 3 these sets are all subvarieties of $T_{t}$.

Now, by Lemma $2, S_{t}$ is finite, hence each element of $S_{t}$ is an irreducible component (in fact an isolated point) of $T_{t}$. So $\left|S_{t}\right| \leq\left(\begin{array}{c}t+1 \\ 2\end{array}\right)^{r}$. Now two cases may occur.

If $T_{t}=S_{t}$ then $S_{t^{\prime}}=\emptyset$ for $t^{\prime}=1, \ldots, t-1$ and so $\left|S_{1} \cup \cdots \cup S_{t}\right|=\left|S_{t}\right| \leq$ $\left(\begin{array}{c}t+1 \\ 2\end{array}\right)^{r}$. This certainly implies $(2.12)$.

If $S_{t}$ is strictly smaller than $T_{t}$ then $T_{t} \backslash S_{t}$ has at least one irreducible component. But then $\left|S_{t}\right| \leq\left(\begin{array}{c}t+1 \\ 2\end{array}\right)^{r}-1$. In conjunction with the induction hypothesis this gives

$$
\begin{aligned}
\left|S_{1} \cup \cdots \cup S_{t}\right| & =\left|S_{1} \cup \cdots \cup S_{t-1}\right|+\left|S_{t}\right| \\
& \leq \sum_{p=1}^{t-1}\left(\begin{array}{c}
p+1 \\
2
\end{array}\right)^{r}-t+2+\left(\begin{array}{c}
t+1 \\
2
\end{array}\right)^{r}-1,
\end{aligned}
$$

which again implies (2.12).

This completes the proof of our induction step, hence of our Proposition.

3. Proof of the Theorem for transcendence degree 1 . We prove the Theorem in the special case that $K$ has transcendence degree 1 over $k$. For convenience we put $N:=\sum_{i=2}^{n+1}\left(\begin{array}{c}i \\ 2\end{array}\right)^{r}-n+1$.

We start with some reductions. There are $\mathbf{a}_{j}=\left(\alpha_{1 j}, \ldots, \alpha_{n j}\right) \in \Gamma(j=$ $1, \ldots, r)$ such that for each $\mathbf{x} \in \Gamma$ there are integers $m, w_{1}, \ldots, w_{r}$ with $m>0$, and $\xi=\left(\xi_{1}, \ldots, \xi_{n}\right) \in\left(k^{*}\right)^{n}$ such that $\mathbf{x}^{m}=\xi \cdot \mathbf{a}_{1}^{w_{1}} \cdots \mathbf{a}_{r}^{w_{r}}$. Let $L$ be the extension of $k$ generated by $a_{1}, \ldots, a_{n}$ and the $\alpha_{i j}(i=1, \ldots, n$, 
$j=1, \ldots, r)$. Then $L$ is the function field of a smooth projective algebraic curve $C$ defined over $k$. Choose $z \in L, z \notin k$, such that the map $z: C \rightarrow$ $\mathbb{P}_{1}(k)=k \cup\{\infty\}$ is unramified at 0 and such that none of the functions $a_{i}, \alpha_{i j}$ has a zero or a pole in any of the points from $z^{-1}(0)$. Thus, $L$ can be embedded into $k((z))$, and the $a_{i}$ and $\alpha_{i j}$ may be viewed as elements of $k[[z]]$ not divisible by $z$. By multiplying the $\alpha_{i j}$ with appropriate constants from $k^{*}$, which we are free to do, we may assume without loss of generality that the $\alpha_{i j}$ belong to $1+z k[[z]]$.

Making the assumptions for the $a_{i}$ and $\alpha_{i j}$ just mentioned, we can apply our Proposition. The functions $\alpha_{i j}^{u}(u \in k)$ are defined uniquely by means of (2.1). Therefore, we can express each $\mathbf{x} \in \Gamma$ as $\xi \cdot \mathbf{a}_{1}^{u_{1}} \cdots \mathbf{a}_{r}^{u_{r}}$ with $u_{1}, \ldots, u_{r}$ in $\mathbb{Q}$ and with $\xi=\left(\xi_{1}, \ldots, \xi_{n}\right) \in\left(k^{*}\right)^{n}$. Putting $\mathbf{A}_{i}:=\left(\alpha_{i 1}, \ldots, \alpha_{i r}\right)(i=$ $1, \ldots, n)$, we can rewrite this as

$$
\mathbf{x}=\left(\xi_{1} \mathbf{A}_{1}^{\mathbf{u}}, \ldots, \xi_{n} \mathbf{A}_{n}^{\mathbf{u}}\right)
$$

with $\xi_{1}, \ldots, \xi_{n} \in k^{*}, \mathbf{u}=\left(u_{1}, \ldots, u_{r}\right) \in \mathbb{Q}^{r}$. Putting in addition $h:=n+1$, $\mathbf{A}_{h}:=(1, \ldots, 1)(r$ times $), a_{h}:=-1, \xi_{h}:=1$ we deduce that if $\mathbf{x} \in \Gamma$ is a non-degenerate solution of (1.1) then

$$
\begin{aligned}
& \sum_{i=1}^{h} \xi_{i} a_{i} \mathbf{A}_{i}^{\mathbf{u}}=0 \\
& \sum_{i \in I} \xi_{i} a_{i} \mathbf{A}_{i}^{\mathbf{u}} \neq 0 \quad \text { for each proper, non-empty subset } I \text { of }\{1, \ldots, h\} .
\end{aligned}
$$

It remains to verify condition (2.4). According to an argument in the proof of Lemma 1 of [5], the set of $\mathbf{u} \in k^{r}$ such that $\left(\mathbf{A}_{i} \mathbf{A}_{h}^{-1}\right)^{\mathbf{u}}=1$ for $i=1, \ldots, h$ is a linear subspace of $k^{r}$, say $V$, which is defined over $\mathbb{Q}$. Now if $\mathbf{u}=$ $\left(u_{1}, \ldots, u_{r}\right) \in V \cap \mathbb{Q}^{r}$, then $\mathbf{A}_{i}^{\mathbf{u}}=1$ for $i=1, \ldots, n$ since $\mathbf{A}_{h}=(1, \ldots, 1)$, and therefore $\mathbf{a}_{1}^{u_{1}} \cdots \mathbf{a}_{r}^{u_{r}}=(1, \ldots, 1)$. This implies $\mathbf{u}=\mathbf{0}$, since otherwise $\operatorname{rank}\left(\Gamma /\left(k^{*}\right)^{n}\right)$ would be smaller than $r$. Hence $V \cap \mathbb{Q}^{r}=\{\mathbf{0}\}$, and therefore $V=\{\boldsymbol{0}\}$ since $V$ is defined over $\mathbb{Q}$. This implies (2.4).

As observed above, if $\mathbf{x} \in \Gamma$ is a non-degenerate solution of (1.1), then $\mathbf{u}$ satisfies (3.2), (3.3), which means that $\mathbf{u}$ belongs to the set $S$ given by (2.5), (2.6). So by the Proposition, we have at most $N$ possibilities for $\mathbf{u}$. Then according to (3.1), the non-degenerate solutions $\mathbf{x}$ of (1.1) lie in at most $N$ $\left(k^{*}\right)^{n}$-cosets. This completes the proof of our Theorem in the special case where $K$ has transcendence degree 1 over $k$.

4. Proof of the Theorem in the general case. We now prove our Theorem in the general case, i.e., when the field $K$ is an arbitrary transcendental extension of $k$. As before, we define $N:=\sum_{i=2}^{n+1}\left(\begin{array}{l}i \\ 2\end{array}\right)^{r}-n+1$.

There is of course no loss of generality in assuming that $K$ is generated over $k$ by the coefficients $a_{1}, \ldots, a_{n}$ and the coordinates of all elements of $\Gamma$. 
Since $\Gamma$ is assumed to have rank $r$, there are $\mathbf{a}_{1}, \ldots, \mathbf{a}_{r} \in \Gamma$ such that for every $\mathbf{x} \in \Gamma$ there are integers $m, z_{1}, \ldots, z_{r}$ with $m>0$ and $\xi \in\left(k^{*}\right)^{n}$ such that $\mathbf{x}^{m}=\xi \cdot \mathbf{a}_{1}^{z_{1}} \cdots \mathbf{a}_{r}^{z_{r}}$. Hence $K$ is algebraic over the extension of $k$ generated by $a_{1}, \ldots, a_{n}$ and the coordinates of $\mathbf{a}_{1}, \ldots, \mathbf{a}_{r}$. Therefore, $K$ has finite transcendence degree over $k$. We will prove by induction on $d:=\operatorname{trdeg}(K / k)$ that for any group $\Gamma$ with $\operatorname{rank}\left(\Gamma /\left(k^{*}\right)^{n}\right) \leq r$, the non-degenerate solutions $\mathbf{x} \in \Gamma$ of (1.3) lie in not more than $N\left(k^{*}\right)^{n}$-cosets. The case $d=0$ is trivial since in that case $\Gamma=\left(k^{*}\right)^{n}$ and all solutions lie in a single $\left(k^{*}\right)^{n}$-coset. Further, the case $d=1$ has been taken care of in the previous section. So we assume $d>1$ and that the above assertion is true up to $d-1$.

We suppose by contradiction that (1.1) has at least $N+1$ non-degenerate solutions, denoted $\mathbf{x}_{1}, \ldots, \mathbf{x}_{N+1} \in \Gamma$, falling into pairwise distinct $\left(k^{*}\right)^{n}$-cosets. For each such solution $\mathbf{x}_{j}=:\left(x_{1 j}, \ldots, x_{n j}\right)$ and for each nonempty subset $I$ of $\{1, \ldots, n\}$ let us consider the corresponding subsum $\sum_{i \in I} a_{i} x_{i j}$, which we denote by $\sigma_{(j, I)}$. In this way we obtain finitely many elements $\sigma_{(j, I)} \in K$, none of which vanishes, since the solutions are nondegenerate.

Further, let $\mathbf{x}_{u}, \mathbf{x}_{v}$ be distinct solutions, with $1 \leq u \neq v \leq N+1$. Since the solutions lie in distinct $\left(k^{*}\right)^{n}$-cosets, for some $i \in\{1, \ldots, n\}$ the ratio $x_{i u} / x_{i v}$ does not lie in $k$. For each pair $(u, v)$ as above let us pick one such index $i=i(u, v)$ and let us put $\tau_{(u, v)}:=x_{i u} / x_{i v} \in K^{*} \backslash k^{*}$.

We are going to "specialize" such elements of $K$, getting corresponding elements of a field with smaller transcendence degree and obtaining eventually a contradiction. We shall formulate the specialization argument in geometric terms.

Let $\widetilde{K}$ be the extension of $k$ generated by $a_{1}, \ldots, a_{n}$ and by the coordinates of $\mathbf{x}_{1}, \ldots, \mathbf{x}_{N+1}$. Thus $\widetilde{K}$ is finitely generated over $k$. Further, let $\widetilde{\Gamma}$ be the group containing $\left(k^{*}\right)^{n}$ and generated over it by $\mathbf{x}_{1}, \ldots, \mathbf{x}_{N+1}$. Then $\widetilde{\Gamma}$ is a subgroup of $\Gamma \cap\left(\widetilde{K}^{*}\right)^{n}$, and so $\operatorname{rank}(\widetilde{\Gamma}) \leq r$. Now (1.1) has at least $N+1$ non-degenerate solutions in $\widetilde{\Gamma}$ lying in different $\left(k^{*}\right)^{n}$-cosets. By the induction hypothesis this is impossible if $\operatorname{trdeg}(\widetilde{K} / k)<d$. So $\operatorname{trdeg}(\widetilde{K} / k)=d$.

The finitely generated extension $\widetilde{K} / k$ may be viewed as the function field of an irreducible affine algebraic variety $V$ over $k$, with $d=\operatorname{dim} V$. Then each element of $\widetilde{K}$ represents a rational function on $V$. Let us consider irreducible closed subvarieties $W$ of $V$, with function field denoted $L:=k(W)$, with the following properties:

(A) $\operatorname{dim} W=d-1$.

(B) There exists a point $P \in W(k)$ such that each of the (finitely many) elements $a_{i}, x_{i j}$ and $\sigma_{(j, I)}, \tau_{(u, v)}$ constructed above is defined and non-zero at $P$; so the elements induce by restriction non-zero rational functions $a_{i}^{\prime}, x_{i j}^{\prime}, \sigma_{(j, I)}^{\prime}$ and $\tau_{(u, v)}^{\prime}$ in $L^{*}=k(W)^{*}$. 
(C) None of the elements $\tau_{(u, v)}^{\prime}$ lies in $k^{*}$.

We shall construct $W$ as an irreducible component of a suitable hyperplane section of $V$.

To start with, (A) follows from the well-known fact that any irreducible component $W$ of any hyperplane section of $V$ has dimension $d-1$.

Let us analyze (B). Each of the elements of $\widetilde{K}^{*}$ mentioned in (B) may be expressed as a ratio of non-zero polynomials in the affine coordinates of $V$; since these elements are defined and non-zero by assumption, none of these polynomials vanishes identically on $V$, so each such polynomial defines in $V$ a proper (possibly reducible) closed subvariety. Take now a point $P \in V(k)$ outside the union of these finitely many proper subvarieties. For (B) to be satisfied it then plainly suffices that $W$ contains $P$.

Finally, let us look at (C). For each $u, v \in\{1, \ldots, n\}, u \neq v$, let $Z(u, v)$ be the variety defined in $V$ by the equation $\tau_{(u, v)}=\tau_{(u, v)}(P)$. Since $\tau_{(u, v)}$ is not constant on $V$, each component of $Z(u, v)$ is a subvariety of $V$ of dimension $d-1$. Choose now $W$ as an irreducible component through $P$ of the intersection of $V$ with a hyperplane $\pi$ going through $P$, such that $W$ is not contained in any of the finitely many $Z(u, v)$. It suffices e.g. that the hyperplane $\pi$ does not contain any irreducible component of any $Z(u, v)$, and there are plenty of choices for that. (For example, for each of the relevant finitely many varieties, each of dimension $d-1 \geq 1$, take a point $Q \neq P$ in it and let $\pi$ be a hyperplane through $P$ and not containing any of the $Q$ 's. Note that here we use the fact that $d \geq 2$.) Since $P \in W(k)$ and $\tau_{(u, v)}$ is not constantly equal to $\tau_{(u, v)}(P)$ on all of $W$ by construction, the restriction $\tau_{(u, v)}^{\prime}$ is not constant, as required.

Consider now the elements $\mathbf{x}_{j}^{\prime}:=\left(x_{1 j}^{\prime}, \ldots, x_{n j}^{\prime}\right) \in L^{n}, j=1, \ldots, N+1$, where the prime denotes, as before, the restriction to $W$ (which by (B) is well-defined for all the functions in question). Notice that the restriction to $W$ is a homomorphism from the local ring of $V$ at $P$ to the local ring of $W$ at $P$ which is contained in $L$. This homomorphism maps $\widetilde{\Gamma}$ to the group $\Gamma^{\prime}$ containing $\left(k^{*}\right)^{n}$, generated over it by the elements $\mathbf{x}_{1}^{\prime}, \ldots, \mathbf{x}_{N+1}^{\prime}$. Thus, $a_{1}^{\prime}, \ldots, a_{n}^{\prime}$ and the coordinates of the elements from $\Gamma^{\prime}$ lie in $L$. Further, $\Gamma^{\prime}$ is a homomorphic image of $\widetilde{\Gamma}$ which was in turn a subgroup of $\Gamma$; therefore $\operatorname{rank}\left(\Gamma^{\prime} /\left(k^{*}\right)^{n}\right) \leq r$. Since the $\mathbf{x}_{j}$ are solutions of (1.1) in $\widetilde{\Gamma}$, the elements $\mathbf{x}_{j}^{\prime}$ are solutions of $a_{1}^{\prime} x_{1}+\cdots+a_{n}^{\prime} x_{n}=1$ in $\Gamma^{\prime}$. Again by (B), we see that none of the (non-empty) subsums $\sigma_{(j, I)}^{\prime}=\sum_{i \in I} a_{i}^{\prime} x_{i j}^{\prime}$ vanishes, so these solutions are non-degenerate. Finally, by (C), no two solutions $\mathbf{x}_{u}^{\prime}, \mathbf{x}_{v}^{\prime}, 1 \leq u \neq v \leq N+1$, lie in a same $\left(k^{*}\right)^{n}$-coset of $\left(L^{*}\right)^{n}$. Since by (A) the field $L$ has transcendence degree $d-1$ over $k$, this contradicts the inductive assumption, concluding the induction step and the proof. 
5. Proof of the Corollary. We keep the notation and assumptions from Section 1. We consider the non-degenerate solutions $\left(x_{1}, \ldots, x_{n}\right) \in \Gamma$ of (1.1) such that

$$
\left(a_{1} x_{1}, \ldots, a_{n} x_{n}\right) \notin\left(k^{*}\right)^{n} .
$$

We first show that each $\left(k^{*}\right)^{n}$-coset of such solutions is contained in a proper linear subspace of $K^{n}$. Fix a non-degenerate solution $\mathbf{x}=\left(x_{1}, \ldots, x_{n}\right)$ of (1.1) with (5.1). Any other solution of (1.1) in the same $\left(k^{*}\right)^{n}$-coset as $\mathbf{x}$ can be expressed as $\mathbf{x} \cdot \xi=\left(x_{1} \xi_{1}, \ldots, x_{n} \xi_{n}\right)$ with $\xi=\left(\xi_{1}, \ldots, \xi_{n}\right) \in\left(k^{*}\right)^{n}$ and $a_{1} x_{1} \xi_{1}+\cdots+a_{n} x_{n} \xi_{n}=1$. Now the points $\xi \in k^{n}$ satisfying the latter equation lie in a proper linear subspace of $k^{n}$, since otherwise $\left(a_{1} x_{1}, \ldots, a_{n} x_{n}\right)$ would be the unique solution of a system of $n$ linearly independent linear equations with coefficients from $k$, hence $a_{1} x_{1}, \ldots, a_{n} x_{n} \in k$, violating (5.1). But this implies that indeed the $\left(k^{*}\right)^{n}$-coset $\left\{\mathbf{x} \cdot \xi: \xi \in\left(k^{*}\right)^{n}\right\}$ is contained in a proper linear subspace of $K^{n}$.

Now our Theorem implies that the non-degenerate solutions of (1.1) with (5.1) lie in at most $\sum_{i=2}^{n+1}\left(\begin{array}{c}i \\ 2\end{array}\right)^{r}-n+1$ proper linear subspaces of $K^{n}$. Further, the degenerate solutions of (1.1) lie in at most $2^{n}-n-2$ proper linear subspaces of $K^{n}$, each given by $\sum_{i \in I} a_{i} x_{i}=0$, where $I$ is a subset of $\{1, \ldots, n\}$ of cardinality $\neq 0,1, n$. By adding these two bounds our Corollary follows.

\section{References}

[1] E. Bombieri, J. Mueller and U. Zannier, Equations in one variable over function fields, Acta Arith. 99 (2001), 27-39.

[2] J.-H. Evertse and K. Györy, On the numbers of solutions of weighted unit equations, Compos. Math. 66 (1988), 329-354.

[3] J.-H. Evertse, H. P. Schlickewei and W. M. Schmidt, Linear equations in variables which lie in a multiplicative group, Ann. of Math. 155 (2002), 807-836.

[4] J. Mueller, S-unit equations in function fields via the abc-theorem, Bull. London Math. Soc. 32 (2000), 163-170.

[5] U. Zannier, On the integer solutions of exponential equations in function fields, Ann. Inst. Fourier (Grenoble) 54 (2004), 849-874.

Mathematisch Instituut

Universiteit Leiden

Postbus 9512

2300 RA Leiden, The Netherlands

E-mail: evertse@math.leidenuniv.nl
Scuola Normale Superiore Piazza dei Cavalieri 7 56126 Pisa, Italy E-mail: u.zannier@sns.it 